\title{
Rational approach, technique and selection criteria for the treatment of lumbar disc herniation with Regenerative Selective Disc therapy
}

\author{
Calogero Riccardo Buscemi, Andrea Licata \\ BioMedical Clinic, Palermo, Italy
}

\begin{abstract}
Low back pain is one of the most common and important clinical, social, economic, and public health problems affecting the human population worldwide. The mechanism of radicular pain in the lumbar region is multifactorial but is likely due to mechanical and/or inflammatory factors. The natural history of disc herniation tends to be favourable. The Italian Society for Oxygen-Ozone Therapy (SIOOT) and the Italian Society for Spinal Surgery (SICV) guidelines recommend a conservative therapeutic approach. The biological action of medical ozone is still under investigation but some mechanisms of action have been proposed to explain its efficacy in disc herniation treatment: i) reduction of the inflammatory components; ii) hyper-oxygenation of the area of interest; iii) diminishing the size of the herniation; iv) stimulation of the repair process. The primary objective of this pilot study was to compare pain and function scores from patients before and after the treatment with Regenerative Selective Disc. The metrics that were established to define significant improvement were: i) improvement $>1.8$ on the Visual Analogue Scale (VAS) score; ii) improvement of 1 level or more on the modified Macnab criteria; iii) improvement $>15 \%$ on the Oswestry Disability Index (ODI). Primary endpoints of this study were changes in the pretreatment and 1 month post treatment VAS, ODI and Macnab scores of the patients. After treatment results show that the patient population's mean VAS, ODI and modified Macnab scores improved beyond
\end{abstract}

\footnotetext{
Correspondence: Calogero Riccardo Buscemi, BioMedical Clinic, via Nicolò Garzilli 34, 90141 Palermo, Italy.

E-mail: Rickcal78@gmail.com
}

Key words: Regenerative Selective Disc therapy; Oxygen-ozone; Disk herniation; Back pain; No-Dol technique; Tri-ox-pro technique; Det-ox technique; Disk-phoresis technique; Bio-back technique; Discectomy; Discolysis; Neuropathy.

Conflict of interest: the authors declare no potential conflict of interest.

Funding: none.

Received for publication: 24 June 2019

Accepted for publication: 5 July 2019

${ }^{\circ}$ Copyright: the Author(s), 2019

Licensee PAGEPress, Italy

Ozone Therapy 2019; 4:8378

doi:10.4081/ozone.2019.8378

This article is distributed under the terms of the Creative Commons Attribution Noncommercial License (by-nc 4.0) which permits any noncommercial use, distribution, and reproduction in any medium, provided the original author(s) and source are credited. the significant improvement scores. In fact, results showed that the mean patient's population improvement scores were 2.9 for VAS, 22 for ODI and 1.4 for modified Macnab. Additionally, significant improvements were shown for $87 \%$ of the patients for VAS scale ( $>2.0$ ), $80 \%$ of the patients for the ODI scale ( $>15$ points) and $80 \%$ patients for the modified Macnab criteria ( $>1$ point).

\section{Introduction}

Low back pain is one of the most common and important clinical, social, economic, and public health problems affecting the human population worldwide. ${ }^{1}$ In $1.6-43 \%$ of the cases there are associated sciatic symptoms, ${ }^{1}$ which, $90 \%$ of the time, are generated by disk herniation. ${ }^{2}$

The mechanism of radicular pain in the lumbar region is multifactorial but is likely due to mechanical and/or inflammatory factors. There is a component related to disc herniation that causes a direct compression of the nerve root or dorsal root ganglion, and an indirect compression on perineural vessels. Once the disk gets injured, facet capsule, epidural tissue surrounding the nerve root and the nerve root itself get inflamed with production of a cascade of inflammatory mediators, all of which activate afferents, sensitize nociceptors and make the nerve exquisitely sensitive to pressure, generating pain with either gentle manipulation or pressure. ${ }^{2-5}$ Because of this, the inflammatory component of radicular pain allows that a bulging or protruding disk generates pain without a necessary root compression.

The natural history of disc herniation tends to be favourable. Spontaneous regression of disc herniation is seen in two-thirds of cases $^{6}$ and a spontaneous resolution of pain within the acute phase (from six to 12 weeks after pain onset) in $60-80 \%$ of patients. ${ }^{7}$

The Italian Society for Oxygen-Ozone Therapy (SIOOT) and the Italian Society for Spinal Surgery (SICV) guidelines recommend a conservative therapeutic approach, expect for cases where the motor and/or sensory deficit is such as to require an immediate surgical decompression of the nerve root.

Percutaneous techniques minimize the invasive nature of surgery, rendering administration more straightforward and faster while sparing healthy tissues and minimizing surgical complications. ${ }^{8}$

Ozone $\left(\mathrm{O}_{3}\right)$ is a strongly oxidant gas with antiseptic, immunomodulating, analgesic and anti-inflammatory properties. ${ }^{8}$ Oxygen-ozone gas mixture $\left(\mathrm{O}_{2} \mathrm{O}_{3}\right)$ is commonly used in clinical practice, mostly in Europe and Asia, in the treatment of nociceptive-neuropathic pain, in inflammatory and degenerative processes of the muscle-skeletal system and especially in degenerative disc disease and disc herniation. ${ }^{9} \mathrm{O}_{3}$ is administered in the form of $\mathrm{O}_{2} \mathrm{O}_{3}$ at non-toxic concentrations ranging from $1 \mu \mathrm{g}$ to $40 \mu \mathrm{g}$ of $\mathrm{O}_{3}$ per $\mathrm{mL}$ of oxygen, using various percutaneous methods. 
The biological action of medical ozone is still under investigation but some mechanisms of action have been proposed to explain its efficacy in disc herniation treatment.

\section{Reduction of the inflammatory components}

$\mathrm{O}_{3}$ interrupts the self-sustained cycle of the inflammatory cascade by altering the breakdown of arachidonic acid to inflammatory prostaglandins.

\section{Hyper-oxygenation of the area of interest}

Disc herniation impinges on the venous and arterial flow, causing phlebostasis and arteriostenosis, which lead to hypoxemia of the tissues; by applying $\mathrm{O}_{2} \mathrm{O}_{3}$ to the herniated site, oxygen concentration increases.

\section{Diminishing the size of the herniation}

$\mathrm{O}_{3}$ breaks down the glycosaminoglycans chains in the nucleus pulposus and reduces their ability to hold water, thereby shrinking the nucleus and subsequently reducing intradiscal and peri-radicular pressure.

\section{Stimulation of the repair process}

$\mathrm{O}_{3}$ promotes the fibroblastic activity, inducing collagen deposition. ${ }^{10}$

The primary objective of this pilot study was to compare pain and function scores from patients before and after the treatment with Regenerative Selective Disc (RSD).

\section{Materials and Methods}

This prospective pilot study was designed as single arm clinical study. All patients were treated from January 2018 to October 2018.

RSD is not an experimental treatment because every one of the included medical procedures is regularly performed and properly validated.

Informed Consent form was signed by each patient prior to his/her treatment.

Baseline questionnaires were given prior the treatment. Then follow-up questionnaires were given immediately after treatment and one month following the treatment.

The primary goal of the study is to monitor the changes between the pre-treatment and one month post-treatment Visual Analogue Scale (VAS), Oswestry Disability Index (ODI) and the modified Macnab scores of patients.
Sixty patients meeting exclusion-inclusion criteria as described in Table 1 were enrolled for this study.

Ozone was generated from oxygen/ozone generator Medical 95 C.P.S. by Multiossigen S.p.A. (Gorle, BG, Italy).

The concentration of the oxygen-ozone mixture produced by the generator was verified using a calibrated UV ozone analyser.

\section{No-dol technique, epidural injection}

A sterile, 19 gauge, $12 \mathrm{~cm}$ long Thuoy needle was inserted in the intervertebral space of the herniated disk disk hernia with central spine approach. Using spindle liquid technique, the epidural space was identified and $40 \mathrm{mg}$ of triamcinolone and $10 \mathrm{mg}$ of levobupivacaine diluited in $15 \mathrm{~mL}$ of $\mathrm{NaCl} 0.9 \%$ injected. Compressive Medications post-treatment was applicated.

\section{Tri-ox-pro technique, paravertebral ozone injection}

A sterile, 22 gauge, $4 \mathrm{~cm}$ long needle was inserted in the intervertebral space of disk hernia with unilateral paramedian approach. Once the under-muscle fascia had been reached, $10 \mathrm{~mL}$ of mixture of $\mathrm{O}_{2} \mathrm{O}_{3}$ al ozone concentration of 15 microgram $/ \mathrm{mL}$ were introduced.

\section{Det-ox technique, systemic oxygen-ozone therapy}

A sterile iv cannula was inserted in the peripheral vein and connected to a sterile SAN O3 kit for major autohemo infusion with ozone, close circuit phthalates free with anticoagulant inside, $35 \mathrm{~mL}$ ACD-A solution, produced by Gobbi Frattini Paolo srl. $180 \mathrm{~mL}$ of blood was withdrawn in about 10 minutes and subsequently mixed with $180 \mathrm{~mL}$ of oxygen-ozone mixture at ozone concentration of 40 $\mathrm{mcg} / \mathrm{mL}$. Afterwards, the blood was reinfused in about 10 minutes.

\section{Disk phoresis and bio-back technique}

A sterile, 27 gauge, $1 \mathrm{~cm}$ long needle was inserted with bilateral paravertebral approach on the dorsal and lumbar vertebral zone. $3 \mathrm{~mL}$ mixture of oxygen-ozone at $2-5 \mathrm{mcg} / \mathrm{mL}$ ozone concentration was introduced in every injection.

Postural correction. Osteopath handling. Physiatrist handling treatment.

\section{Methods to assess pain relief}

VAS, ODI and modified Macnab criteria questionnaires were used to assess patient pain and function before and 1 month after treatment.

The metrics that were established to define significant improvement were: i) improvement $>1.8$ on the VAS score; ii) improvement of 1 level or more on the modified Macnab criteria; iii) improvement $>15 \%$ on the ODI.

Primary endpoints of this study were changes in the

Table 1. Inclusion and exclusion criteria.

\begin{tabular}{ll} 
Inclusion criteria & Exclusion criteria \\
$\begin{array}{l}\text { 20-70 years of age } \\
\text { Single herniated disk between L3 and S1 evident on MRI }\end{array}$ & $\begin{array}{l}\text { Previous spine surgery evident on MRI } \\
\text { Abnormal neurological exam indicative of cord compression or cauda equina } \\
\text { syndrome }\end{array}$ \\
\hline Able to return for 1-month follow-up evaluation & $\begin{array}{l}\text { Spondylolisthesis, vertebral canal stenosis or disc hernation }>5 \text { mm evident } \\
\text { on MRI } \\
\text { Lower back pain and/or sciatica exacerbated by sitting and/or standing }\end{array}$ \\
\hline No previous disc surgery & $\begin{array}{l}\text { Infection as evidenced by patient clinical evaluation, history and blood test } \\
\text { Platelet count }<60,000, \text { INR }>1.5, \text { PTTT }>40 \text { sec }\end{array}$ \\
\hline- & Disc height loss $>75 \%$ evident on MRI
\end{tabular}


pretreatment and 1-month post treatment VAS, ODI and Macnab scores of the patients.

\section{Regenerative Selective Disc method}

Preliminary clinic control with analysis of Magnetic Resonance Imaging and electromyography data.

Explanation of treatment plan. Informed Consent.

Duration of the therapeutic path: 6-8 weeks.

2 sessions each week.

First week: NO-DOL therapy with epidural technique if VAS of patient is $>7$. Tri-ox-pro Technique with paravertebral ozone injection.

Second week: Tri-ox-pro Technique with paravertebral ozone injection. Det-ox Technique with systemic oxygen-ozone therapy.

Third week: Tri-ox-pro Technique with paravertebral ozone injection. Disk phoresis and bio-back technique.

Fourth week: Tri-ox-pro Technique with paravertebral ozone injection. Det-ox Technique with systemic oxygen-ozone therapy.

At the end of fourth week, if the VAS pain scale, ODI and Macnab criteria were improved the treatment continued regularly.

If VAS pain scale, ODI and Macnab criteria were not improved a NO-DOL therapy with epidural technique was performed again.

Fifth week: Tri-ox-pro Technique with paravertebral ozone injection. Disk phoresis and bio-back technique.

Sixth week: Tri-ox-pro Technique with paravertebral ozone injection. Det-ox Technique with systemic oxygen-ozone therapy.

Seventh week: Tri-ox-pro Technique with paravertebral ozone injection. Disk phoresis and bio-back technique.

Eighth week: Tri-ox-pro Technique with paravertebral ozone injection. Disk phoresis and bio-back technique.

\section{Results}

Only six patients at the beginning had VAS $>7$, so only six patients received an epidural technique during the first week.

After treatment results show that the patient population's mean VAS, ODI and modified Macnab scores improved beyond the significant improvement scores.

In fact, results showed that the mean patient's population improvement scores were 2.9 for VAS, 22 for ODI and 1.4 for modified Macnab.

Additionally, significant improvements were shown for $87 \%$ of the patients for VAS scale $(>2.0), 80 \%$ of the patients for the ODI scale ( $>15$ points) and $80 \%$ patients for the modified Macnab criteria ( $>1$ point).

Only three patients needed a second epidural treatment after four weeks, $50 \%$ less than first week. Two of them underwent a surgery discectomy at the end of the treatment.

Two patients did not finish the treatment and underwent surgical discectomy between the third and the seventh week.

Three patients underwent surgical discectomy after the end of the treatment.

Two patients at the end of the treatment did not improve enough to obtain the goal but refused surgery.

\section{Discussion}

Oxygen-ozone treatment has been used extensively for relieving lower back pain. There were no adverse events associated with ozone injections.
The mechanism of pain relief for this study has a joint action because four treatments were involved: i) No-dol technique: median epidural injection of $40 \mathrm{mg}$ of triamcinolone, $10 \mathrm{mg}$ of levobupivacaine, diluted in $15 \mathrm{~mL}$ of $\mathrm{NaCl} 0.9 \%$; ii) Tri-ox-pro Technique: paravertebral injection of $10 \mathrm{~mL}$ of mixture of oxygenozone with $15 \mathrm{mcg} / \mathrm{mL}$ of ozone concentration; iii) Det-ox Technique: systemic oxygen-ozone therapy; iv) Disk phoresis and bio-back technique.

We believe that primary mechanism of action of the oxygenozone mixture is reduction in size of the herniated disc due to a redux reaction between the ozone and the glycosaminoglycans in the nucleus pulposis. This reduces the osmotic gradient across the end plates resulting in disc dehydration and volume reduction. The ozone decreases the disc pressure on the nerve via nucleus pulposus reduction and steroid reduces inflammation in the area adjacent to the disc.

Systemic oxygen-ozone therapy has an anti-inflammatory, anti-edema systemic effects and neurotrophic role improving nerve function.

Disk phoresis and bio-back technique reduced muscle paravertebral contraction, improved the biomecanic posture and improved the intervertebral distance reducing the disc pressure.

\section{Conclusions}

The study showed that patients achieved significant improvements in VAS, ODI and modified Macnab scales for pain and function, without adverse events, after receiving RSD therapy for the treatment of herniated disc. Conclusions about the long term safety and efficacy of RSD therapy for the treatment of herniated disc are not warranted by this study alone because of the lack of control group and short follow-up times. RSD is a medical treatment of herniated disc that while avoiding the risks and complications of surgery achieved significant improvements of VAS, ODI and modified Macnab scales at least $87 \%$ of patients treated.

\section{References}

1. De Oliveira Magalhaes F, Dotta L, Sasse A, et al. Ozone therapy as a treatment for low back pain secondary to herniated disc: A systematic review and meta-analysis of randomized controlled trials. Pain Physician 2012;15:E115-29.

2. Crockett MT, Moynagh M, Long N, et al. Ozone-augmented percutaneous discectomy: A novel treatment option for refractory discogenic sciatica. Clin Radiol 2014;69:1280-6.

3. Buric J, Rigobello L, Hooper D. Five and ten year follow-up on intradiscal ozone injection for disc herniation. Int J Spine Surg 2014;1:8.

4. Li B, Xu X, Du Y, et al. CT-guided chemonucleolysis combined with psoas compartment block in lumbar disc herniation: A randomized controlled study. Pain Med 2014;15:1470-6.

5. Murphy K, Muto M, Steppan J, et al. Treatment of contained herniated lumbar discs with ozone and corticosteroids: A pilot clinical study. Can Assoc Radiol J 2015;66:337-84.

6. Splendiani A, Perri M, Conchiglia A, et al. MR assessment of lumbar disk herniation treated with oxygen-ozone diskolysis: The role of DWI and related ADC versus intervertebral disk volumetric analysis for detecting treatment response. Neuroradiol J 2013;26:347-56. 
7. Borrelli E. Mechanism of action of oxygen ozone therapy in the treatment of disc herniation and low back pain. Acta Neurochir Suppl 2011;108:123-5.

8. Lu W, Li YH, He X. Treatment of large lumbar disc herniation with percutaneous ozone injection via the posterior-lateral route and inner margin of the facet joint. World $\mathrm{J}$ Radiol 2010;2:109-12.
9. Zhang Y, Ma Y, Jiang J, et al. Treatment of the lumbar disc herniation with intradiscal and intraforaminal injection of oxygenozone. J Back Musculoskelet Rehabil 2013;26:317-22.

10. Muto M, Giurazza F, Silva RP, Guarnieri G. Rational approach, technique and selection criteria treating lumbar disk herniations by oxygen-ozone therapy. Interv Neuroradiol 2016;22:736-40. 